\title{
Drug Resistance Pattern of Mycobacterium Tuberculosis Isolated from Patients Attending a Referral Hospital
}

\author{
A B M Abdul Wadud ${ }^{1}$, A S M Matiur Rahman ${ }^{2}$, Md Ruhul Amin Miah ${ }^{3}$, Ahmed Abu Saleh ${ }^{3}$ \\ ${ }^{1}$ Department of Microbiology \& Immunology, LABAID LTD, Dhaka , ${ }^{2}$ Armed Forces Institute of Pathology, Dhaka Cantonment, \\ ${ }^{3}$ Dept of Microbiology \& Immunology, BSMMU, Dhaka .
}

\begin{abstract}
This study was carried out to isolate $M$. tuberculosis from smear positive sputum samples by MB/BacT automated culture system and investigate the drug resistance pattern. Among 101 smear positive cases 96 (95.05 $\%)$ yielded growth of Mycobacteria, $3(02.97 \%)$ were contaminated and $2(01.98 \%)$ showed no growth. Culture positive detection time for $M$. tuberculosis was lowest 7 days and highest 36.3 days and average detection time was11.3 days. Detection time for NTM was 19.2 days .Among 96 isolates 95 (98.96\%) were $M$. tuberculosis and remaining $1(1.04 \%)$ was photochromogen Non-tuberculous Mycobacteria (NTM). Out of 95 M. tuberculosis 51 $(53.84 \%)$ were resistant to at least one drug, $38(40.00 \%)$ to INH, $31(32.63 \%)$ to RMP, 13(13.68 \%) to SM, 11 $(11.58 \%)$ to EMB and $10(10.53 \%)$ to PZA and multi-drug resistant tuberculosis (MDR-TB) was 21 (22.11 $\%)$. Resistance to one drug was $21(22.11 \%)$, two drugs $15(15.79 \%)$, to three drugs $10(10.53 \%)$ and resistant to four or more drugs $5(5.26 \%)$ cases.
\end{abstract}

\section{Introduction:}

Tuberculosis is a disease of great antiquity and caused more suffering and death than any other bacterial infections ${ }^{1}$. It causes one billion deaths in the last 200 years $^{2}$. Deaths from tuberculosis comprise $25 \%$ of all avoidable deaths in the developing countries. $95 \%$ of tuberculosis cases and $98 \%$ of tuberculosis deaths are in developing countries; $75 \%$ of these cases are in the economically productive age group (15-50 yrs). ${ }^{3}$

Tuberculosis is one of the major health problems in Bangladesh. Recent estimate suggest that $2.3 \%$ of the population of Bangladesh get infected with tuberculosis every year, and about 300000 people progress to develop disease

\section{Correspondence:}

Dr. A B M Abdul Wadud

Department of Microbiology

\& Immunology, LABAID LTD, Dhaka annually. About half of these have infectious pulmonary tuberculosis and continue to spread the disease. In 1996 a total of 63,000 cases of tuberculosis were registered by the National Tuberculosis Program (NTP), representing 21\% of the estimated incidence ${ }^{6}$.

Globally, the emergence of multidrug resistant strains of Mycobacterium tuberculosis is an increasing problem which adversely affects patient care and public health. In contrast to other bacteria, resistance of $M$. tuberculosis is exclusively associated with chromosomal mutation. Resistance of $M$. tuberculosis to anti-tuberculosis drugs is the result of a spontaneous genetic event and, worse, "a man-made amplification of the natural phenomenon" 6 . The global magnitude of drug resistance tuberculosis is not well described. A worldwide survey was performed by World Health Organization/ International Union against Tuberculosis and Lung Disease (WHO/IUATLD) global surveillance project between 1994 to 1997 revealed drug resistances varied from $2.3 \%$ to $42.4 \%$. Rates of drug resistance to isoniazid range from $2.3 \%$ to $39.0 \%$, 
sterptomycin $0.9 \%$ to $35.1 \%$, rifampicin $0 \%$ to $23 \%$, ethambutal $0 \%$ to $9.4 \%$ and multi drug resistance was $0 \%$ to $22.1 \%{ }^{7}$. Recently ICDDR'B, Dhaka has reported resistance to any drug was $48.4 \% 8$.

\section{$M$ aterials and methods}

A total of 101 smear positive cases of both sexes, age varied from 06 to 68 years, irrespective of history of taking antitubercul osis drug are included in this study. Patients were selected from National TB Control Center, Shaymoli, Dhaka, Institute of Disease of the Chest and Hospital, Mohakhali, and Combined Military hospital (CMH), Dhaka, between February 2003 and January 2004. History relevant to tuberculosis such as time and duration, regime, place or centers of treatment was recorded in predesigned Data sheet.

Three consecutive moming sputum samples from each patient were collected in properly labeled screw cap disposable plastic bottles after oral gurgling with normal water. All samples were carried to Department of Microbiology, Armed Forces institute of Pathology (AFIP), Dhaka, where further processing was done. Sputum samples were processed and stained by Ziehl-Neelsen (Z-N) method and examined for Acid-Fast Bacilli (AFB). One sputum sample from each smear positive patient was processed, inoculated in MB/BacT automated culture bottle contain Middlebrook 7H9 broth and incubated in automated culture system (MB/BacT automated culture system, Organon Teknika, USA) at $37^{\circ} \mathrm{C}$ for six weeks according to the manufacturer's instructions.

The preliminary identification of mycobacterium isolate depends on their growth in MB/BacT bottle and detected and displayed on monitor and growth rate. Specific identification is accompl ished by the performance of Z-N stain and battery of biochemical tests.

Sensitivity tests against Isoniazid (INH), Rifampicin (RMP), Streptomycin (SM), Ethambutol (EMB), and Pyrazinamide (PZA) were done by proportion method on LowensteinJensen (L-J) media.

All inoculated L-J drug and control media were incubated at $3^{\circ} \mathrm{C}$ for 3 weeks. The media were examined at 48 hours then weekly. The reading for drug susceptibility were taken at 3 weeks as after that drug deterioration may allow growth of susceptible organisms. The reading of growth on control and drug containing media were recorded according to Kent and Kubicall. Drug resistance was expressed in Proportion Method, where a strain is considered to be drug resistance if the number of colonies that grow on a drug containing media is $1 \%$ or more of the colonies that grow on a control drug free media. The control (drug free) media must show good growth at least 50 to 150 colonies. M. tuberculosis H37Rv strain used as a control strain.

\section{Results}

A total of 101 sputum smear positive pul monary tuberculosis patients were studied. Among 101 cases $73(72.28 \%)$ were male and remaining $28(27.72 \%)$ were female. The ratio of male and female was 2.61:1. Majority, 76 (75.25\%) of them were in the age group of 15 to 44 years with peak incidence in 25-34 years of age 35 (34.65\%).

Of the 101 cases majority of them 79 (78.22\%) were in lowincome group and only 22 (21.78\%) from middle-income group. No patient was from upper-income group. Majority, 62 (61.39\%) of the patients came from urban area .

Of these 101 smear positive cases, culture for Mycobacteria were positive in $96(95.05 \%)$ cases, contamination in 3 (2.97\%) and no growth of Mycobacteria in 2 (1.98\%) cases (Table-l).

Table - I: Results of culture on MB/BacT automated culture system among

$(n=101)$ smear (AFB) positive sputum specimens.

\begin{tabular}{lcl}
\hline Results of culture & Number & Percentages \\
\hline Growth of Mycobacteria & 96 & 95.05 \\
Contamination & 3 & 02.97 \\
No growth of Mycobacteria & 2 & 01.98 \\
Total & 101 & 100 \\
\hline
\end{tabular}

Culture positive detection time for $M$. tuberculosis was lowest 7 days and highest 36.3 days and average detection time was11.3 days. Detection time for NTM was 19.2 days .

Among these 96 culture positive cases 95 (98.96\%) were identified as $M$. tuberculosis and rest 1 (1.04\%) was nontubercul ous mycobacteria (NTM) which was photochromogen (Table ll) .

Table-II: Distribution of $M$. tuberculosis and Nontuberculous mycobacteria among the isolated Mycobacteria. $(\mathrm{n}=96)$

\begin{tabular}{llc}
\hline Mycobacteria & Number & Percentages \\
\hline M. tubercul osis & 95 & 98.96 \\
$\begin{array}{l}\text { Non-tuberculous mycobacteria } \\
\text { ( Photochromogen ) }\end{array}$ & 1 & 1.04 \\
Total & 96 & 100 \\
\hline
\end{tabular}


Identification tests for Mycobacterium isolates were done in accordance with the standard procedures and among the 95 M. tuberculosis strains all were slow grower, positive for Niacin test, Nitrate reduction test. They failed to grow on PNB containing media and negative for catalase test The single NTM isolate was a slow grower, produced visible colonies on PNB containing media, positive Catal ase test and negative for Niacin and Nitrate reduction test, which was photochromogen.

Table III shows the sensitivity pattern of 95 strains of $\mathrm{M}$. tuberculosis to 5 antituberculosis drugs. Out of these 95 strains 44 (46.32\%) were sensitive to all 5 drugs and 51 (53.84 \%) strains were resistant to one or more drugs. Highest resistance (40\%) was found in $\mathrm{INH}$ either alone or in combination with other drugs.

Table-III: Sensitivity pattern of M. tuberculosis to five Anti-tuberculosis

method. $(n=95)$

drugs in L-J media by proportion

\begin{tabular}{lll}
\hline Name of drugs & $\begin{array}{l}\text { No. of sensitive } \\
\text { strains (\%) }\end{array}$ & $\begin{array}{l}\text { No. of resistant } \\
\text { strains (\%) }\end{array}$ \\
\hline Isoniazid & $57(60.00)$ & $38(40.00)$ \\
Rifampicin & $64(67.68)$ & $31(32.63)$ \\
Streptomycin & $82(86.32)$ & $13(13.68)$ \\
Ethambutol & $84(88.42)$ & $11(11.58)$ \\
Pyrazinamide & $85(89.47)$ & $10(10.53)$ \\
Sensitiveto all drugs & $44(46.32)$ & - \\
Resistanceto any drug & - & $51(53.68)$
\end{tabular}

From resistance pattern of 51 strains of $M$ tuberculosis it appears that 21 (41.18\%) were resistant to a single drug, 15 (29.41 \%) were resistant to 2 drugs, 10 (19.61 $\%)$ resistant to 3 drugs, 4 (7.84 \% ) were resistant to 4 drugs and only one case was found resistant to 5 drugs. MDR was found in 21 (41.18\%) cases. (Table IV ) .
Table- IV : Resistance pattern of 51 drug resistant strains of M. tuberaulosisto 5 antituberculosis drugs.

\begin{tabular}{|c|c|c|c|}
\hline $\begin{array}{l}\text { Number } \\
\text { of drugs }\end{array}$ & $\begin{array}{l}\text { Name } \\
\text { of drugs }\end{array}$ & $\begin{array}{l}\text { No. of } \\
\text { Resistant } \\
\text { strains (\%) }\end{array}$ & $\begin{array}{l}\text { Total } \\
\text { (\%) }\end{array}$ \\
\hline 1 drug & $\begin{array}{l}\text { Isoniazid (INH) } \\
\text { Rifampicin(RMP) } \\
\text { Streptomycin (SM) } \\
\text { Etharnbutol(EMB) }\end{array}$ & $\begin{array}{c}10(19.61) \\
8(15.69) \\
1(1.96) \\
2(3.92)\end{array}$ & $21(22.11)$ \\
\hline 2 drugs & $\begin{array}{l}\text { *INH+RMP } \\
\text { INH+SM } \\
\text { INH+PZA } \\
\text { INH+EMB } \\
\text { EMB+SM } \\
\text { RMP+PZA }\end{array}$ & $\begin{array}{l}7(13.73) \\
2(3.92) \\
2(3.92) \\
2(3.92) \\
1(1.96) \\
1(1.96)\end{array}$ & 15(15.79) \\
\hline 3 drugs & $\begin{array}{l}* I N H+R M P+S M \\
* I N H+R M P+E M B \\
* N H+R M P+P Z A \\
\text { INH+SM+EMB }\end{array}$ & $\begin{array}{l}5(9.80) \\
2(3.92) \\
2(3.92) \\
1(1.96)\end{array}$ & 10(10.53) \\
\hline 4 drugs & $\begin{array}{l}\text { *INH+RMP+EMB+PZA } \\
\text { *INH+RMP+SM+EMB }\end{array}$ & $\begin{array}{l}3(5.88) \\
1(1.96)\end{array}$ & $4(4.21)$ \\
\hline 5 drugs & *INH+RMP+SM+EMB+PZA & $1(1.96)$ & $1(1.05)$ \\
\hline$* \mathrm{MDR}$ & & $21(22.11)$ & \\
\hline
\end{tabular}

INH-Isoniazid, RMP-Rifampicin, SM-Streptomycin, PZAPyrazinamide, EMB -Ethambutol

*MDR: Multi-drug resistant: Resistance to both I soniazid and Rifampicin with or without resistance to other drugs.

\section{Discussion}

In this study drug resistance of Mycobacterium tubercul osis to at least one drug were found in $53.68 \%$ cases. The situation is highly al arming. Highest resistances (40.00\%) were found in INH which is the most popular drugs, followed by RMP (32.63\%) cases. Resistances to SM werefound in (13.68 \%) cases, to EMB (11.58 \%) and to PZA (10.53\%) cases. The efficiency of current tubercul osis control program in any country is assayed by drug resistant pattem. ICDDR'B, Dhaka reported resistance to any drug was $48.4 \%$, resistance to INH, RMP, SM and EMB was $17.4 \%, 7.4 \%, 45.3 \%$ and $9.9 \%$ respectively ${ }^{8}$. Similarly Riantawan et al from Thailand, reported acquired drug resistance in $53.7 \%$ cases of tuberculosis, and resistance to INH was $29.2 \%$, to RMP $43.0 \%$, to SM $21.1 \%$ and to EMB $4.8 \%$ and MDR was 
$25.2 \%$ cases $^{10}$. Friden et al from New York also reported similar type of result where acquired drug resistance in $44 \%$ cases of tuberculosisil.

Lina et al from Bombay (India) reported drug resistance to INH, RMP, SM, EMB and MDR-TB was $30.41 \%, 58.55 \%$, $46.95 \%, 3.67 \%$ and $25.25 \%$ respectively ${ }^{12}$. A similar study from Haryana, India shows MDR-TB of the same order (24 \%) 30. Trivedi and Desai from Gujrat (India) reported acquired drug resistance to INH, RMP and SM in 55.8\%, $37.3 \%$ and $26.9 \%$ respectively ${ }^{31}$.

Some countries like New Zealand and Botswana has a very low drug resistant cases, $4.8 \%$ respectively, while countries like Domonican Republic, Russia and Latvia has a higher rate $42.4 \%, 38.3 \%$ and $41.6 \%$ respectively 9 . Effective tuberculosis control and management program in developed country like USA, UK, France has a favourable effect on the low incidence of drug resistance, while our tuberculosis control and management program are as effective as our neighbour country and thereby the resistance pattern remain similar in these countries.

In this study highest drug (40.00\%) resistance was found against INH either al one or in combination with other drugs. INH resistance is important, because it is a potent bactericidal drug, and is an important component of short course antitubercul osis regimen. It al one may kill $90 \%$ of the bacillary population in a patient's lesions during the first few days of chemotherapy ${ }^{16}$. So, resistance against INH may leads to treatment failure. This high rate of $\mathrm{INH}$ resistance might be due to wide use of this drug in first line treatment of patients and irregular and inadequate treatment.

Rifampicin was found resistant $32.63 \%$ cases in this study. Rifampicin - the most important drug in DOTS program - is also gradually becoming ineffective due to development of resistance. As Rifampicin is a potent bactericidal and sterilizing drug which act on dormant and persister bacilli on short exposure and resistance to Rifampicin may lead to the failure of DOTS program ${ }^{17}$. The reason for high resistance to Rifampicin might be due to irregular use due to its high cost, using Rifampicin in other condition like Leprosy, Leishmaniasis and PUO. National tuberculosis programme and patient compliance may al so have some role.

Drug resistance against Streptomycin (SM) was found 13.68 $\%$ in this study. SM is less potent bactcricidal drug and introduced first for the treatment of tuberculosis. The relatively less SM resistance than INH and RMP might be due to decrease use of it It is no longer used in standard regimen but in retreatment cases.

Out of 95 isolated strains , 11 (11.58 \%) were resistant to EMB. It is bacteriostatic drug but in large dose can act as bactericidal ${ }^{16}$. Resistance to PZA was found in $10.53 \%$ cases in our study. PZA susceptibility test is difficult to perform as it acts at lower $\mathrm{PH}^{\mathrm{H}}$ where bacteria could not grow. So far that reason, $\mathrm{PH}^{\mathrm{H}}$ of the drug media must be adjusted at $5.5^{11}$. It was not suggested to do drug resistance surveillance against PZA but can be performed in small scale for special reason ${ }^{18}$.

Pyrazinamide (PZA) is a less potent bactericidal drug. It is most important sterilizing drugs because of their ability to kill semi-dormant bacilli capable of surviving bactericidal action of INH and so of giving rise to relapse after treatment. The bacilli specifically killed by PZA are probably those with their metabolism inhibited by an acid environment such as in macrophages and in areas of acute inflammation ${ }^{16}$.

In this study drug resistance of $M$. tubercul osis to single drug was found in $22.11 \%$ cases, to two drugs in $15.79 \%$, to three drugs in $10.53 \%$ and to four drugs in $4.21 \%$ cases. One strain was resistant to all the five drugs. In Bangladesh, Van Deun et al found 21 . 95\% strains were resistant to one drug, $13 \%$ resistant to two drugs, $10.5 \%$ to three drugs and $8.5 \%$ to four or more drugs ${ }^{19}$. Resistances against two or three drugs are difficult to treat and often result in treatment fail ure ${ }^{20}$.

In the present study $22.11 \%$ strains of $M$. tubercul osis were Multi-drug resistance tuberculosis (MDR-TB). MDR-TB is usually a death sentence in developing countries as it is an incurable disease for anyone who does not get access to the most sophisticated and expensive heal th care Similar rate of multi-drug resistance also reported from Argentina (22.2\%), Dominican Republic (19.7\%), Spain (20.5\%), higher than these rate were found $54.4 \%$ in Latvia21. Similar rate of MDR-TB was reported from India $25.25 \% 12$. From Thailand, Riantawan et al reported 25.2\% MDR among acquired drug resistance cases ${ }^{10}$. Lower rate $5.56 \%$ was reported by Van Duen et al and $6.8 \%$ by ICDDR'B from Bangladesh19. 8.

The regimen for treatment of tubercul osis has changed very little during last decades; combination drug therapy is the main stream of treatment. Afford have been limited only to changing this combination. Since the methodology of drug susceptibility for Mycobacteria is cumbersome, tedious and takes weeks to come to a conclusion. So almost similar regimen is prescribed for every tuberculosis patient irrespective of extent of the lesion or the sensitivity pattern of causative strains. Until new drugs are available for treatment of tuberculosis, present regimen will be rendered ineffective by development of resistance, so combination therapy is inevitable for days to come till new more efficient drugs are available in future.

An easy, fast and efficient method for drug susceptibility to M. tuberculosis may change the situation. The proportion method is widely used throughout the world for it is inexpensive, comparison to radiometric method and other molecular methods. A part from cost the wide spectrum use of 
radiometric material for drug sensitivity test may lead to new problem, particularly its disposal.

\section{Conclusion}

The present study creates the impression that fairly high rate of anti-tuberculosis drug resistance among the tuberculosis patients and also high MDR-TB. By taking history of previous treatment for tuberculosis, drug resistance patients group and new cases from same family should be identified. Susceptibility test of these groups should be performed with all commonly used first line anti-tuberculosis drugs, and then appropriate drugs should be sel ected for curative treatment to prevent spread of drug resistant tuberculosis. Our priority is not the management, but the prevention of MDR tuberculosis.

\section{R eferences:}

1 Myers, JA. Captain of All These Men of Death. Tuberculosis Historical Highlight Warrem H.Green, St Louis. Cited from Clinical Tuberculosis (ed) Davies PDO, Chapman \& Hall Medical, 1994.1: 1-15.

2. Ryan LW, Arathoon E and Loverde YD. The epidemiologic pattern of Drug Resistant Mycobacterium tuberculosis infections: A community Respir Dis 1989: 139: 1282-1285.

3. World Health Organization. Guidelines for the management of Drug-Resistant Tuberculosis. WHO 1997. whqlibdoc.who.int/hq/1997/WHO_TB_96.210_(Rev.1).pdf

4. World Health Organi sation; TB death reach historic levels. Press releaseWHO/22, 21 Mar 1996A: 1-3

5. World Health Organisation. Review of the National tuberculosis programme of Bangladesh, 1997. WHO/TB/1998A; 238: 1-48.

6. Ramswamy S, Musser JM. Molecular genetic basis of antimicrobial agent resistance in M. tuberculosis: 1998 update. Tuberc Lung Dis 1998; 79: 3-29.

7. World Health Organisation. Anti-tuberculosis Drug Resistance WHO/IUATLD Global Project on Anti-tuberculosis Drug Resistance Surveillance. Geneva, Switzerland, 1997. WHO / TB/ 97.229.

8. ICDDR'B. Health and science bulletin Nov 2002. Vol. 1, no. 1; 6-10.

9. Kent PT and Kubica GP. Public Health Mycobacteriology: A Guide for the level III laboratory. US Department of Health and
Human Services. Centre for Disease control and Prevention, Atlanta, Georgia 30333. 1985; p. 159-184.

10. Riantawan P, Punnotok L,Chaisuksuwan R,Pransujarti V. Resistance of Mycobacteriub tuberculosis to antituberculosis drugs in the Central Region of Thalind, 1996; Int J Tuberc Lung Dis 1998; 2(8): 616-620.

11. Friden TR, S'terling T, Pablos-mandez A, Kilburn JO, Couthen GM and Dooley SW. The Emergence of drug resistant tubercul osis in NewYork City. N EngJ Med 1993; 328: 521-6.

12. Lina D, Priya M and Sweta C. Drug Resistance in tuberculosis. Bombey Hospital Journal Apr 1999; www.bhj.org/joumal/1999_4103_july99/original_253.htm

13. JanmejaAK and Raj B. Acquired drug resistance in tubercul osis in Hariana, India, JAPI 1998; 46: 23-31.

14. Trivedi SS and Desai SO. Primary antituberculosis drug resistance and acquired rifampicin resistance in Gujrat, India. Tubercle 1988; 69: 379-82.

15. World Health Organisation. Anti-tubercul osis Drug Resistance in the world. The WHO/IUATLD Global Project on Antituberculosis Drug Resistance Surveillance. Geneva, Switzerland, 1997. WHO / TB/ 97.229.

16. International Union Against Tuberculosis and Lung Disease Anti tuberculosis regimens of chemotherapy. Bulletin IUATLD, 1988; 63:64.

17. Huq AKMS, Khan ABMBR, Sabur SAMA, Azad A and Miah NU. Study of case finding for pulmonary tuberculosis in out patient departments of general hospitals Bangladesh. Chest and Heart Bulletin 1995: 25-30.

18. World Health Organisation: Guidelines for surveillance of drug resistance in tubercul osis 1997. WHO/TB/1996 B; 216:1-36.

19. Van Duen A, Aung KJM, Chowdhury S, et al. Drug susceptibility of Mycobacterium tuberculosis in a rural area of Bangladesh and its relevance to the national treatment regimens. IntJ Tuberc Lung Dis 3(2): 1999; 143-148.

20. World Health Organization. Guidelines for the management of Drug-Resistant Tuberculosis. WHO 1997. whqlibdoc.who.int/hq/1997/WHO_TB_96.210_(Rev.1).pdf

21. Pablos-Mendez A, Raviglione MC, Laszlo A, et al. Global surveillance for antituberculosis drug resistance, 1994-1997. NEJ M 1998; 338: 1641-49. 